论 文

\title{
HIV-1 病毒样颗粒疫苗的构建及免疫原性研究
}

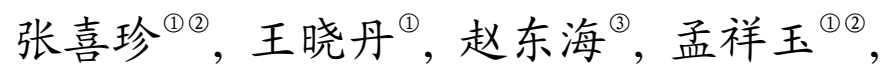 \\ (1) 吉林大学生命科学院, 艾滋病疫苗国家工程实验室, 长春 130012; \\ (2) 吉林大学生命科学院, 分子酶学教育部重点实验室, 长春 130012; \\ (3) 吉林医药大学病理学教研室, 长春 132013 \\ * 联系人, E-mail: xianghui@jlu.edu.cn; weikong@jlu.edu.cn \\ 收稿日期: 2011-05-25; 接受日期: 2011-10-25 \\ 国家自然科学基金(批准号: 30371317)资助项目
} 赵兴红 ${ }^{(1)}$ (2) 千湘晖 ${ }^{\text {(1) (2) }}$, 孔维 ${ }^{11(2) *}$

\begin{abstract}
摘要人免疫缺陷病毒 HIV 在全球的迅猛传播使得有效疫苗的研制工作变成重中之重。 出于安全考虑, 减毒或灭活的 HIV 病毒不能作为疫苗应用, VLPs 则因为不具有病毒基因 组而带来的安全性成为一个极具吸引力的选择. 本研究通过共转染笁选得到了一个有效 而持久表达 HIV-1 结构蛋白 $g a g$ 和 $e n v$ 的真核细胞系, 证实了细胞培养上清中的 $g a g$ 和 $e n v$ 能够自组装成为病毒颗粒. 这些 VLPs 能够在不经佐剂加强的条件下诱导产生具有特异性 的体液和细胞免疫应答.
\end{abstract}

关键词

HIV-1

共转染

稳定表达细胞系 病毒样颗粒(VLPs)

疫苗
随着人类免疫缺陷病毒的不断传播, 研发一种 安全、有效、经济的疫苗成为当务之急. 要设计出一 株有效的疫苗面临 3 个问题: 病毒的变异性、免疫逃 逸和适当的评估免疫的动物模型 ${ }^{[1]}$. 为了克服免疫逃 逸, 一个主要的办法就是要找到 HIV-1 蛋白的保守区, 例如结合到 CD4 细胞和 CCR 5, CXCR4 上的外壳蛋白 $g p 120$ 的保守位点, 或者是另一个外壳蛋白 $g p 41$ 的复 合受体结合位点 ${ }^{[2 \sim 5]}$. HIV-1 病毒的变异性可以通过 宿主免疫系统诱导中和抗体而得到解决. 设计出一 个预防性病毒样颗粒疫苗恰好能使上述 2 个问题得 到解决 ${ }^{[6]}$. HIV-1 VLPs 是以 gag 蛋白为基础构建出一 个复合物结构, 这种结构能够自我组装成特定的-形 态和大小都相似的不成熟 HIV-1 颗粒. 由于没有感染 性, 导致复制缺陷, VLPs 疫苗比传统的灭活疫苗和 减毒疫苗更具安全性. VLPs 不同的形式使它有着独 特的性质 ${ }^{[7 \sim 10]}$. 只有包装过的 VLPs 才能在表面表
达全长的结构蛋白, 尽量模仿原有的外壳三聚体结 构 ${ }^{[7,11,12]}$. 对于不成熟的 HIV-1 病毒相似的抗原免疫 系统有良好的应答 ${ }^{[13]}$. VLP 抗原可以通过 MHC-I 和 MHC-2 途径被加工成递呈抗原, 诱导 CD4 和 CD8 介 导的免疫应答 ${ }^{[14 \sim 16]}$. 上述情况表明, VLPs 即能够高 效地诱导体液免疫, 也能高效地诱导细胞免疫. 当务 之急就是要设计出一种预防性的 HIV 疫苗, 在 HIV 病毒感染的初级阶段进行阻断, 从这方面来说, VLPs 疫苗是一个非常有前景的重组疫苗.

目前, HIV-1 VLPs 疫苗正处于研制阶段 ${ }^{[12,17 ~ 22] . ~}$ VLPs 是现在最具潜力的候选疫苗, 但是它的免疫原 型仍有待提高. 本研究利用 2 种质粒共转染 293 细 胞, 获得高效持续表达结构蛋白 $g a g$ 和 $e n v$ 的真核细 胞系. 其分泌的病毒样颗粒疫苗在不添加任何佐剂 的情况下就可以诱导特异性的体液免疫和细胞免疫 应答. 


\section{1 材料与方法}

\section{1 材料}

pcDNA3.1(-)质粒、lipofectmin-2000 购于 Invitrogen 公司. D-GPEi, pcDNA 3.1 EnvB/C, pNL43.Luc.R-E 质粒和 HOS-CD4-CCR5、293 细胞等为 本实验室保存. 用购于 Qiagen 的小提试剂盒提取 质粒, 终产物不含内毒素(<2.5 U/mg). HIV-1 阳 性血. 清来自广西 AIDS 病人. 抗人 HRP-IgG 购于 Jackson-Immuno 研究室, INC 和 G418 购于 Invitrogen 公司.

\section{2 重组 293 细胞和 HIV-VLP 的构建与表达}

D-GPEi 和 pcDNA3.1(-)共转染 293 细胞获得同 时表达 HIV-1 结构蛋白 Gag 和 Env 的重组 293 细胞 系. D-GPEi 含有 CMV 启动子, kanamycin 抗性基因, 原核细胞高拷贝因子和内含子 A. HIV-1 中国流行株 的主要结构蛋白 gagpol 和 env 分别克隆进 Gag 融合 和 Pol 的 $\mathrm{N}$ 末端表达载体的 2 个表达框 ${ }^{[23]}$. 质粒的 所有组装均符合美国 FDA 的临床实验安全管理标 准, 并能够稳定高效的表达外源基因. 293 细胞培养 用含 $10 \%$ 小牛血清的 DMEM 基本培养基培养 (HyClone Laboratories). D-GPEi 和 pcDNA3.1(-)通 过 lipofectmin-2000 共转染 293 细胞. 转染后 $48 \mathrm{~h}$ 用 胰酶消化重悬并系列稀释细胞, 培养基中加入 G418 抗生素使细胞回贴. 重组表达的 293 细胞在含有浓 度为 $1 \mathrm{mg} / \mathrm{mL} \mathrm{G} 418$ 的培养基中生长. 2 3 周后收获 单克隆细胞并用 Western blot 检测表达情况. 收集阳 性克隆细胞的培养上清, $4000 \mathrm{r} / \mathrm{min}$ 离心 $30 \mathrm{~min}$ 去除 细胞碎片, 离心后的上清液用 $0.22 \mu \mathrm{m}$ 膜过滤. 滤过 后的上清液在 $4{ }^{\circ} \mathrm{C}$ 条件下用 Beckman 超速离心机 SW28 转头 $26000 \mathrm{r} / \mathrm{min} 30 \%$ 庶糖垫, $1.5 \mathrm{~h}$ 离心 2 次, 沉淀收获后用免疫电子显微镜及 Western blot 检测 分析.

\section{3 免疫水平检测}

选 6 8 周龄的雌性 BALB/c 小鼠, 每组 6 只, 通 过小鼠尾静脉注射分别给予提纯后的 VLPs 0.5, 2.5, 12.5 和 $25 \mu \mathrm{g} 4$ 个不同剂量和 $100 \mu \mathrm{g}$ DNA 进行免疫. 对照组鼠注射去除内毒素的 PBS. 免疫方案以 3 针为 基础, 初免后第 3 周和第 5 周给予加强免疫. 所有免
疫方案均不加佐剂.

\section{4 样品收集处理}

最后 1 针免疫后 1 周尾静脉取血收集单只动物血. 样, 用标准方法收获血清后 $-80^{\circ} \mathrm{C}$ 保存备用.

\section{5 透射电子显微镜检测}

病毒样颗粒通过铜网液滴化处理, $5 \mathrm{~min}$ 后用滤 纸出去多余的液滴, 用 JEOL-8100 型电子显微镜在 $200 \mathrm{kV}$ 条件下进行透射电子显微镜分析.

\subsection{CTL 检测}

第 4 次免疫后 2 周取脾, 用在 $1 \mathrm{mmol} / \mathrm{L}$ 的合成 小肽 $37^{\circ} \mathrm{C}$ 罦育 $2 \mathrm{~h}$ 条件下激活的 $1 \times 10^{6}$ 个 P815 细胞 作为靶细胞. 细胞毒性实验通过 CTL 试剂盒 (Promega)用标准的检测乳酸脱氢酶释放测定法完成.

\subsection{ELISPOT 实验}

小鼠脾内的 HIV-1 特异性 $\mathrm{T}$ 细胞作用也可以用 ELISPOT 实验通过检测 HIV-1 抗原诱导 IFN- $\gamma$ 的产 生而间接检测出来, 结果根据鼠 IFN- $\gamma$ ELISPOT 试 剂盒(BD) 说明书来操作完成. 用 $100 \mu \mathrm{L} 5 \mu \mathrm{g} / \mathrm{mL}$ 抗 -IFN- $\gamma$ 多克隆抗体预先包被底部为硝酸纤维素膜的 96 孔板并置于 $4^{\circ} \mathrm{C}$ 过夜, $37^{\circ} \mathrm{C}$ 封闭 $1 \mathrm{~h}$. 分离后提纯 后的脾细胞以一定浓度加到 96 孔板内, 每浓度 3 复 孔, 并加入小肽 $(\mathrm{P} 7)$ 刺激细胞, 96 孔板置于 $37^{\circ} \mathrm{C}$ 睬育 $24 \mathrm{~h}$. 弃去孔内液体, 洗板, 加入生物素化的抗 -IFN- $\gamma$ 抗体 $37^{\circ} \mathrm{C}$ 孵育 1.5 h. $1 / 1000$ 稀释的辣根过 氧化物酶(HRP)标记的抗体孵育 $1 \mathrm{~h}$, 加入 $\mathrm{HRP}$ 底物 $30 \mathrm{~min}$ 后显色, 流水洗去染色液, 晾干, 计数阳性 斑点.

\section{8 中和抗体检测}

pcDNA 3.1 EnvB/C 和 pNL4-3.Luc.R-E 质粒共转 染 293T 细胞. 转染后 $48 \mathrm{~h}$ 收集细胞上清液, 上清液 终浓度分别为 $1: 1,1: 2,1: 4$ 和 $1: 8$ 与血清样本于 $37^{\circ} \mathrm{C}$ 共孵育 $1 \mathrm{~h}$, 混合孵育后的液体通过 DEAE 感染 表达 CD4-CXCR4 的 HOS 细胞. $72 \mathrm{~h}$ 收获 HOS 细胞, 裂解, 通过化学发光检测系统检测 (Promega Inc. USA), 化学发光的表达通过定量以相对光单位通过 Dynex MLX 化学发光检测仪完成, 中和抗体的抑制 浓度通过线性回归曲线分析计算, 中和抗体的浓度 
指的是混合孵育前的浓度.

\section{9 统计学分析}

方差分析值用 SPSS 13.0 软件得出. $P<0.05$ 表明 结果存在显著差异. 所有数据在标准误差范围内.

\section{2 结果}

\subsection{HIV-1 gagPol, env 基因在 293 细胞的有效表达 和 HIV-1 VLPs 的获得}

D-GPEi 和 pCDNA 3.1 质粒共转染重组的 293 细 胞, pCDNA 3.1 具有带篎选标记的 G418 抗性基因, 能够增加目的基因的整合效率.

为用 pcDNA3.1(-)载体成功的构建表达目的基 因的稳定表达细胞系, 本研究选择 D-GPEi 和 pcDNA3.1 的比例为 3:1 共转染 293 细胞, $48 \mathrm{~h}$ 更换 加入 G418 的选择培养基, 并笁选表达 HIV-1 结构蛋 白 gagpol 和 $e n v$ 的阳性克隆细胞, 3 周后 Western blot 检测(结果未显示). 为了检测阳性克隆细胞蛋白的分 泌, 细胞上清中通过 $30 \%$ 蔗糖垫密度离心, 沉淀用 PBS 重悬, 并通过 SDS-PAGE 和 Western Blot 检测 (图 1).

\section{2 细胞表达的稳定性}

为检测细胞表达的稳定性, 将篮选的阳性克隆 细胞持续培养 12 个月, 培养 1 个月和 12 个月的细胞 裂解产物均检测到 HIV-1 gagpol 和 env 蛋白的表达, 结果表明, 目标蛋白能够在细胞内持久稳定的表达. 在稳定表达的细胞上清中也可以检测到 HIV-1VLP 的分泌(图 2).

\subsection{HIV-1 VLPs 的组装}

为研究 HIV-1 gagpol 和 env 蛋白的合成与组装, 收集阳性细胞上清, 通过 10\% 40\%蔗糖密度梯度, $4^{\circ} \mathrm{C}$ 条件下, $26000 \mathrm{r} / \mathrm{min}$ 离心 $4 \mathrm{~h}$, 通过低密度分泌至 40\%和 30\%密度层, 免疫印迹结果表明, 蛋白能够组 装成病毒样颗粒(VLP), 颗粒中包含 HIV-1 结构蛋白 gagpol 和 env(图 3).

\subsection{VLPs 的形态学分析}

HIV-1 VLPs 的分子量太大不能用非变型聚丙烯

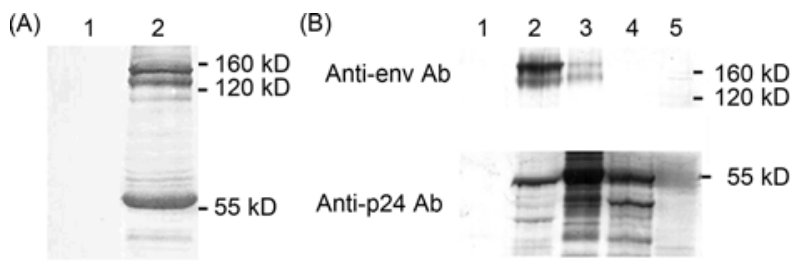

图 1 病毒样颗粒的产生和分析通过材料方法中描述的免 疫蛋白印迹分析

(A) 提纯后的 VLP 的与 HIV-阳性血清作用的免疫印记结果. 1: 空 白细胞上清; 2: 提纯后的 VLP. (B) 阳性细胞与提纯后的分别与 P24 单抗和多克隆 $g p 120$ 抗体作用后的免疫印迹结果. 1: 空白; 2 4: 不同阳性克隆

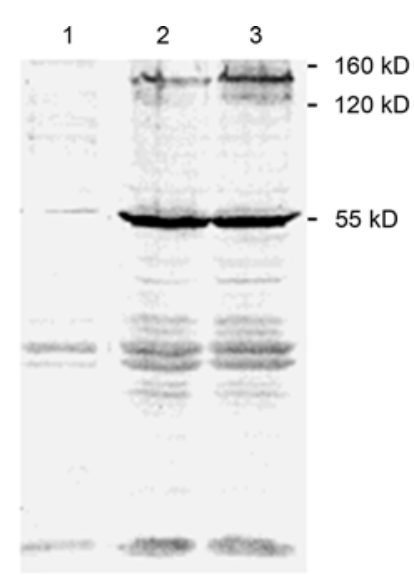

图 2 持续培养 12 个月的阳性细胞裂解物的免疫印迹分析 1: 空白细胞裂解; 2: 培养 1 个月细胞裂解产物; 3 : 培养 12 个月细 胞裂解产物

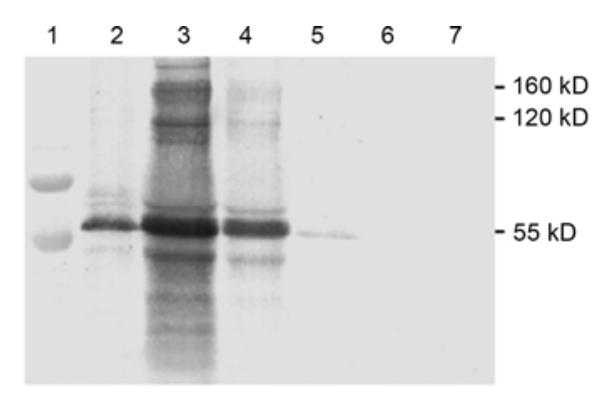

图 3 VLPs 的梯度分析阳性克隆细胞上清通过 $30 \%$ ４0\% 密度梯度蔗糖垫

$\mathrm{sw} 28$ 转头在 $4^{\circ} \mathrm{C}$ 条件下 $26000 \mathrm{r} / \mathrm{min}$ 离心 $4 \mathrm{~h}$ 从底部到顶部收集 离心产物(从左到右)蛋白电泳后用抗 HIV-1 阳性血清作用后免疫印 迹分析. 1: Marker; 2: 底部沉淀; 3 6: 40\% 10\% 梯度层; 7: 上清层

酰胺凝胶电泳或者 MALDI-TOF 飞行质谱分析. 为证 实 HIV-1 结构蛋白 $g a g$ 和 $e n v$ 确实组装成了病毒样颗 粒, 通过透射电子显微镜观测阳性细胞培养上清液 
通过 $30 \%$ 蔗糖垫超速离心后的样品. 数据结果表明, 结构蛋白组装成了直径大约 $100 \mathrm{~nm}$ 的与天然病 毒大小相近的病毒样颗粒(图 4).

\subsection{HIV -1 特异的抗体反应}

免疫后血清抗体的特异性通过免疫印迹检测. 如图 5 所示, 疫苗免疫后引起了特意的抗体反应, 没 有其他的免疫原性产生也没有免疫原性的改变. 2.5 25 $\mu \mathrm{g}$ VLPs 产生的 HIV-1-Gag 特异的抗体反应 比 $100 \mu \mathrm{g}$ DNA 抗体强.

\subsection{HIV-1 特异的 CTL 反应}

病毒样颗粒将通过 MHC I 类途径被抗原提呈细 胞(APCs)提呈给 $\mathrm{CD} 8^{+} \mathrm{T}$ 细胞产生细胞毒性反应 ${ }^{[14]}$. 为检测 $\mathrm{T}$ 细胞反应强弱, 以 3 针的免疫程序分别给予 小鼠免疫 VLPs 不同剂量 $(0.5,2.5,12.5$ 和 $25 \mu \mathrm{g})$ 和 DNA $100 \mu \mathrm{g}$. 最后一针免疫 1 周后, 通过 P815/ $\mathrm{BALB} / \mathrm{c}$ 靶细胞杀伤实验作为 HIV-1 特异性的 $\mathrm{CD} 8^{+} \mathrm{T}$ 细胞体外评价. $0.5 \mu \mathrm{g}$ VLPs 组和 DNA 组 CTL 反应低, 随着注射剂量的增加反应增强. CTL 反应以 $12.5 \mu \mathrm{g}$ 剂量组为最强, $25 \mu \mathrm{g}$ VLPs 组与之比较反应没有明显

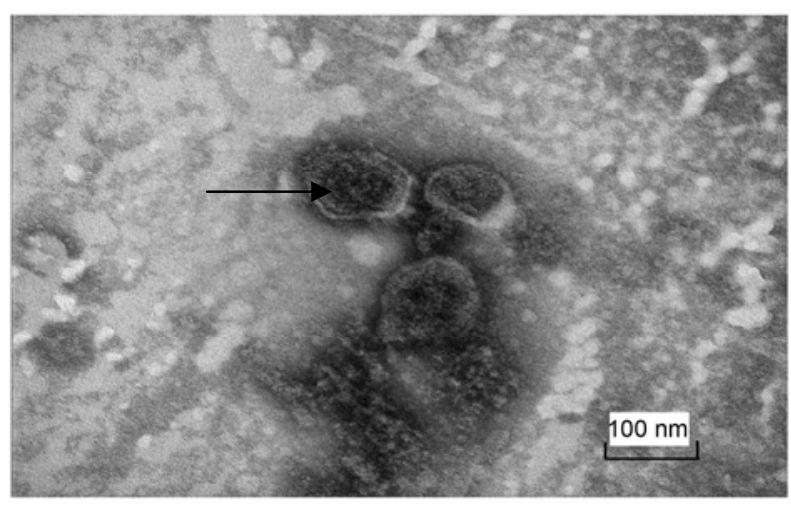

图 4 VLP 的透射电子显微镜结构

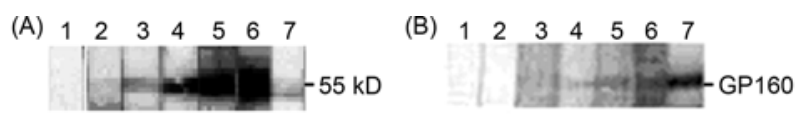

图 5 免疫鼠血清的抗体的 HIV-1 Gag 和 Env 蛋白的免疫 印记检测

收集 3 针免疫后的鼠血清 1:20 稀释(6 只鼠为一组). (A) 1: PBS 对照 组; lane 2 5: VLP 0.5, 2.5, 12. 5 和 $25 \mu \mathrm{g}$; 6: 阳性对照组; 7: DNA $100 \mu \mathrm{g}$. (B) 1: PBS 对照组; 2: DNA $100 \mu \mathrm{g} ; 3 \sim 6$ : VLP 0.5, 2.5, 12.5 和 $25 \mu \mathrm{g}$; 7: 阳性对照组
改变, 因此认为 $12.5 \mu \mathrm{g}$ VLPs 即可引起足够的 CTL 反应(图 6), 并且 CTL 的抗原表位主要存在于 GAG 的 NC 区(p7). 在 BALB/c 免疫保护的有效性的不能 给予评价.

\subsection{IFN $-\gamma$ ELISPOT 实验表明 VLPs 免疫能够引 起 HIV-1 特异的 CTL 反应}

在自然感染阶段, HIV-1 特异的 Th1 细胞、CTL 作用以及抗病毒的细胞因子 (IFN- $\gamma$, TNF- $\alpha$, IL-2) 在对 抗病毒方面起主要作用 ${ }^{[24]}$. ELISPOT 实验检测 HIV-1 特异的 $\mathrm{CD}^{+} \mathrm{T}$ 细胞分泌 IFN- $\gamma$ 的分泌量. $25 \mu \mathrm{g}$ 剂量 和 $12.5 \mu \mathrm{g}$ 剂量的 VLPs 产生的免疫效果相同, 0.5 和 $2.5 \mu \mathrm{g}$ 剂量的免疫都不如 $12.5 \mu \mathrm{g}$. 这表明 $12.5 \mu \mathrm{g}$ 已 经能够足够来诱导细胞免疫了(图 7).

\subsection{VLPs 诱导产生中和抗体与抗体水平}

pNL4-3.Luc.R-E 质粒包含苂光素酶和 HIV-1 全 套基因组, 除了 env 的移码突变. $293 \mathrm{~T}$ 细胞能够通过 共转染包含 $e n v$ 基因的 pNL4-3.Luc.R-E 质粒和 pcDNA 3.1 EnvB/C 质粒, 表达 HIV-1 结构蛋白 $g a g$ 和 $e n v$, 这 2 种蛋白能被整合到病毒颗粒中, 使颗粒 也包含苂光素. 颗粒被称作假病毒, 没有复制性, 但 仍然能够感染 HOS-CD4-CCR5 细胞, 感染水平能够 通过苂光素酶含量被检测到. 中和作用可以通过血. 样与转染后的细胞上清共孵育后的荧光素酶含量值 变化而测出. 结果表明, $0.5 \mu \mathrm{g}$ 的 VLPs 就可以诱导 出中和抗体. 抗体水平随免疫剂量的增加而增加. 结果还表明, 抗体也能够中和假病毒的感染性. 中 和抗体的结合表位主要在 Pr55 和 gp120 上. 诱导抗

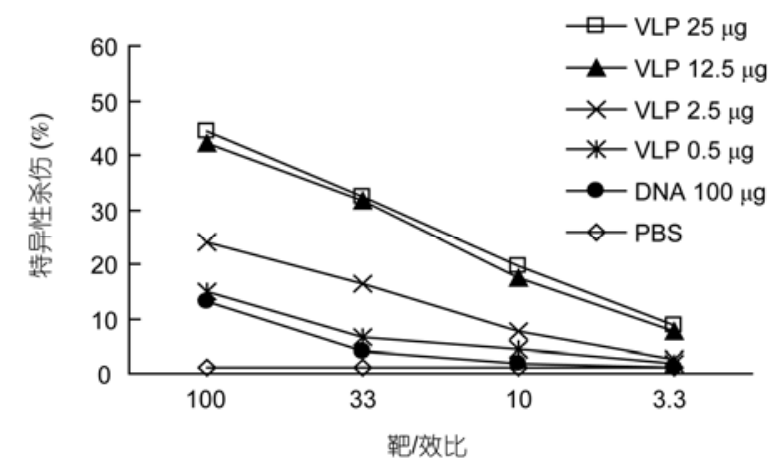

图 6 收集免疫鼠的脾细胞进行细胞毒性分析 用小肽刺激的 P815 细胞作为靶细胞, 通过乳酸脱氢酶的释放情况 检测特异的细胞裂解 
体最多的 VLPs 剂量为 12.5 和 $2 \mu \mathrm{g}$, 并且 DNA 引 起的中和抗体水平较所有剂量的 VLPs 都低, 此结果 表明, VLP 免疫诱导中和抗体的能力强与 DNA, 并且 它的最佳剂量为 $12.5 \mu \mathrm{g}$. 血清 $1: 2$ 和 $1: 4$ 倍稀释后抗 体的中和活力下降, 说明中和抗体是 HIV-1 特异的 (图 8).

\section{3 讨论}

与减毒活疫苗相比, VLPs 疫苗的主要优势是 VLP 能够表达多种抗原表位, 能够刺激机体产生多 样化的免疫应答, 并且没有减毒活疫苗那样的负面 影响. VLP 有潜力激发出体内和体外两种抗原递呈方 式, 能够通过 MHC-1 途径和 MHC-2 途径进行抗原递 呈. 这种多抗原表位疫苗比单一组分疫苗更能产生 广泛的免疫应答, 并且能够清除 HIV-1 的逃逸突变
体. VLPs 疫苗在将来的一期临床试验中比多种单一 疫苗共同注射更经济便宜. 比起单独的重组蛋白疫 苗的另一个优点就是结合进入细胞后的表达适当受 体的能力好. HIV-1 VLPs 疫苗能够通过 gp 120 结合到 CD4 和趋化因子受体上进而进入到专门的抗原递呈 细胞当中, 比如巨噬细胞和树突状细胞当中 (两种细 胞型都表达 CD4 和 CCR5).

病毒蛋白在感染之后能够被加工和递呈到 MHC I 分子, 因此, 促进 $\mathrm{T}$ 细胞的 APCs 递呈. 另外, 结合到 抗体上的 VLPs 能够通过 Fc 受体被巨噬细胞吞噬掉, 因此, 又增加了 MHC II 途径的抗原递呈.

VLPs 已经在杆状病毒和酵母的系统中表达 过 ${ }^{[2527]}$. 这些系统能够从真核细胞中产生出大量的 颗粒. 但它们都受一定的限制只能表达单一的基因, 不易用来生产多基因的 VLPs. 另外, 要使颗粒从酵 母中出芽, 外膜也需要被移除掉.

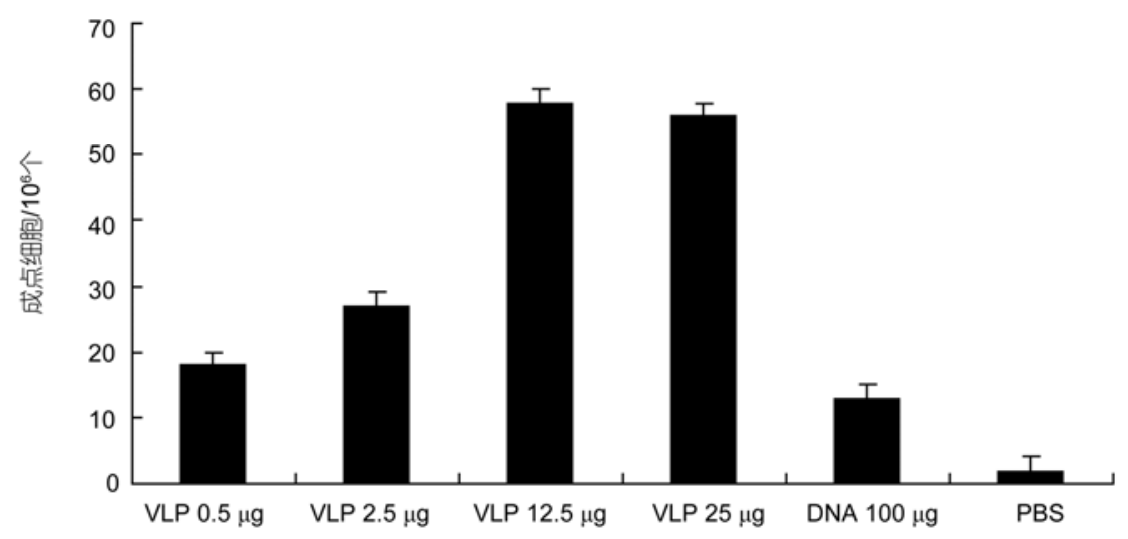

图 7 VLP 疫苗和 DNA 疫苗肌肉注射后, 脾细胞分泌出 IFN-r 每个柱形条代表每 $10^{6}$ 个细胞内分泌的 IFN-r 量

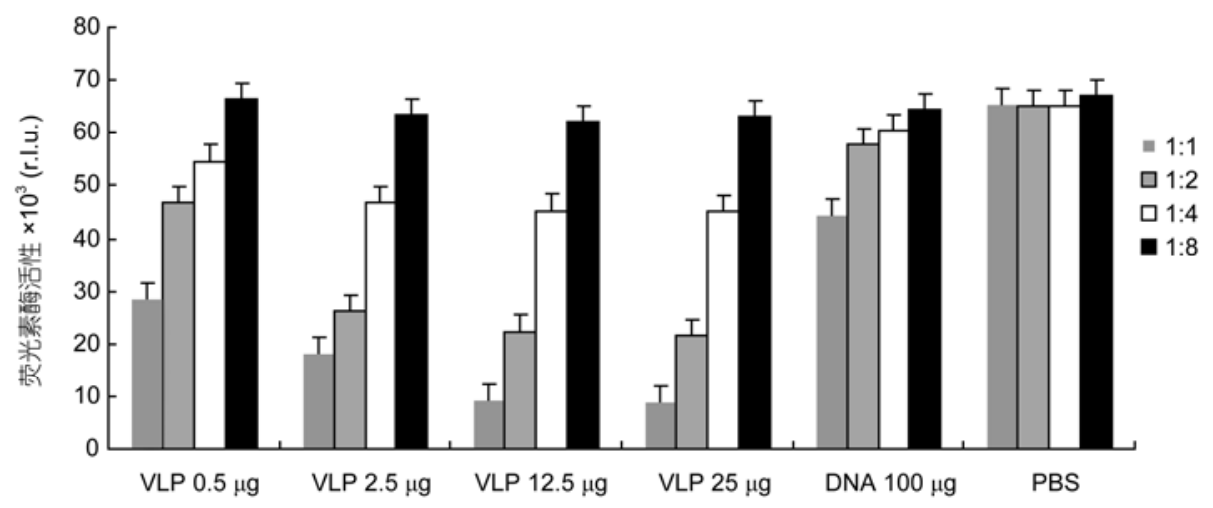

图 8 假病毒感染 HOS-CD4-CCR5 细胞后苂光素酶终点检测

共转染细胞上清和血清样本共同孵育感染细胞. 结果表明检测到了细胞内苂光素酶的表达. 该数据是通过 3 次平行实验得出的结果 
为克服上述不足, 本研究首先利用 2 种质粒进行 共转染, 用新需素作为稳定篮选标记, 最终笁选出了 能够高效稳定表达主要结构蛋白 gagpol 和 env 的真 核细胞系. $g a g$ 蛋白可以自动组装到 VLPs 中, 而 $e n v$ 蛋白的存在可以提供更多的抗原决定簇 ${ }^{[22]}$, 于是可 以利用 $g a g$ 蛋白的自组装结构和共表达的 $g a g$ 和 $e n v$ 来生产 VLPs 颗粒. 实验证明, 这个细胞系不仅生产
出的 VLPs 性质稳定, 数量也多.

通过本研究的方法生产的 VLPs 不带病毒的核酸 是安全的, 还能诱导出强的体液免疫和细胞免疫, 为 HIV-1 疫苗提供了广阔的应用前景. 由于表达的 gagpol 和 env 具有与野生 HIV-1 病毒相似的结构, 要 确定这个细胞系是否是最佳的候选疫苗, 接下来仍需 做许多工作, 以便为将来的优化提供更可靠的证据.

\section{致谢 感谢于晓方教授对本研究的建议和技术上的指导.}

\section{参考文献}

1 Gamble L J, Matthews Q L. Current progress in the development of a prophylactic vaccine for HIV-1. Drug Des Devel Ther, 2010, 5: 9-26

2 Fenouillet E, Barbouche R, Jones I M. Cell entry by enveloped viruses: redox considerations for HIV and SARS-coronavirus. Antioxid Redox Signal, 2007, 9: 1009-1034

3 Devico A, Fouts T, Lewis G K, et al. Antibodies to CD4-induced sites in HIV gp120 correlate with the control of SHIV challenge in macaques vaccinated with subunit immunogens. Proc Natl Acad Sci USA, 2007, 104: 17477-17482

4 Doms R W, Moore J P. HIV-1 membrane fusion: Targets of opportunity. J Cell Biol, 2000, 151: F9-F14

5 Wyatt R, Sodroski J. The HIV-1 envelope glycoproteins: Fusogens, antigens, and immunogens. Science, 1998, 280: 1884-1888

6 Tagliamonte M, Tornesello M L, Buonaguro F M, et al. Conformational HIV-1 envelope on particulate structures: a tool for chemokine coreceptor binding studies. J Transl Med, 2011, 9(Suppl 1): S1

7 Buonaguro L, Buonaguro F M, Tornesello M L, et al. High efficient production of Pr55(gag) virus-like particles expressing multiple HIV-1 epitopes, including a gp120 protein derived from an Ugandan HIV-1 isolate of subtype A. Antiviral Res, 2001, 49: 35-47

8 Gheysen D, Jacobs E, D E Foresta F, et al. Assembly and release of HIV-1 precursor Pr55gag virus-like particles from recombinant baculovirus-infected insect cells. Cell, 1989, 59: 103-112

9 Yamshchikov G V, Ritter G D, Vey M, et al. Assembly of SIV virus-like particles containing envelope proteins using a baculovirus expression system. Virology, 1995, 214: 50-58

10 Wagner R, Deml L, Fliessbach H, et al. Assembly and extracellular release of chimeric HIV-1 Pr55gag retrovirus-like particles. Virology, 1994, 200: 162-175

11 Wang B Z, Liu W, Kang S M, et al. Incorporation of high levels of chimeric human immunodeficiency virus envelope glycoproteins into virus-like particles. J Virol, 2007, 81: 10869-10878

12 Crooks E T, Moore P L, Franti M, et al. A comparative immunogenicity study of HIV-1 virus-like particles bearing various forms of envelope proteins, particles bearing no envelope and soluble monomeric gp120. Virology, 2007, 366: 245-262

13 Yao Q, Bu Z, Vzorov A, et al. Virus-like particle and DNA-based candidate AIDS vaccines. Vaccine, 2003, 21: 638-643

14 Bachmann M F, Lutz M B, Layton G T, et al. Dendritic cells process exogenous viral proteins and virus-like particles for class I presentation to $\mathrm{CD}^{+}$cytotoxic T lymphocytes. Eur J Immunol, 1996, 26: 2595-2600

15 Deml L, Kratochwil G, Osterrieder N, et al. Increased incorporation of chimeric human immunodeficiency virus type 1 gp120 proteins into Pr55gag virus-like particles by an Epstein-Barr virus gp220/350-derived transmembrane domain. Virology, 1997, 235: 10-25

16 Reimann J, Schirmbeck R. Alternative pathways for processing exogenous and endogenous antigens that can generate peptides for MHC class I-restricted presentation. Immunol Rev, 1999, 172: 131-152

17 Visciano M L, Diomede L, Tagliamonte M, et al. Generation of HIV-1 Virus-Like Particles expressing different HIV-1 glycoproteins. Vaccine, 2011

18 Speth C, Bredl S, Hagleitner M, et al. Human immunodeficiency virus type-1 (HIV-1) Pr55gag virus-like particles are potent activators of human monocytes. Virology, 2008, 382: 46-58

19 Deml L, Speth C, Dierich M P, et al. Recombinant HIV-1 Pr55gag virus-like particles: potent stimulators of innate and acquired immune responses. Mol Immunol, 2005, 42: 259-277 
20 Doan L X, Li M, Chen C, et al. Virus-like particles as HIV-1 vaccines. Rev Med Virol, 2005, 15: 75-88

21 Lebedev L R, Karpenko L I, Poryvaeva V A, et al. Design of virus-like particles, exposing HIV-1 epitopes. Mol Biol(Mosk), 2000, 34: 480-485

22 Young K R, Mcburney S P, Karkhanis L U, et al. Virus-like particles: designing an effective AIDS vaccine. Methods, 2006, 40: 98-117

23 姜春来. 细胞因子对 HIV-1 疫苗 DNA prime/MVA boost 的加强作用. 长春: 吉林大学, 2005

24 Seder R A, Hill A V. Vaccines against intracellular infections requiring cellular immunity. Nature, 2000, 406: 793-798

25 Wagner R, Fliessbach H, Wanner G, et al. Studies on processing, particle formation, and immunogenicity of the HIV-1 gag gene product: a possible component of a HIV vaccine. Arch Virol, 1992, 127: 117-137

26 Sakuragi S, Goto T, Sano K, et al. HIV type 1 Gag virus-like particle budding from spheroplasts of Saccharomyces cerevisiae. Proc Natl Acad Sci USA, 2002, 99: 7956-7961

27 Tsunetsugu-yokota Y, Morikawa Y, Isogai M, et al. Yeast-derived human immunodeficiency virus type 1 p55(gag) virus-like particles activate dendritic cells (DCs) and induce perforin expression in Gag-specific CD8(+) T cells by cross-presentation of DCs. J Virol, 2003, 77: $10250-10259$ 